Title

Equity in the health systems of middle income countries: a review or reviews

\title{
Research question
}

What are the mechanisms and interactions between actors that affect equity in the health systems of middle-income countries?

\section{Search terms}

MeSH: Health Equity (Opportunity to attain full health potential and no one is disadvantaged from achieving this potential because of their social position or other socially determined circumstance. Year introduced: 2016)

\section{Searches}

PubMed, Scopus, Web of Science, Ovid and Embase databases were used, to search with MeSH terms and with DeCS terms BVS was used.

A search was made with keywords (MeSH and DeCs terms in Spanish and English) as follows:

MeSH: Health Equity (Opportunity to attain full health potential and no one is disadvantaged from achieving this potential because of their social position or other socially determined circumstance. Year introduced: 2016)

DeCS: Health Equity, Equity, Social Inequity, Health Status Disparities, Review, Systematic Review

\section{Search equations}

("Health Equity" OR "Health Services Accessibility" OR "Delivery of Health Care" OR "Health Services Administration") AND ("Systematic Review")

"health equity"[All Fields] OR "equity"[All Fields] OR "inequality"[All Fields] OR "inequalities"[All Fields] OR "disparities"[All Fields] AND "Systematic Review"[All Fields]

(("Healthcare Disparities"[Mesh] AND "Socioeconomic Factors"[Mesh]) AND "Health Equity/organization and administration"[Mesh]) AND ( "Systematic Review" [Publication Type] OR "Book Review" [Publication Type] OR "Systematic Reviews as Topic"[Mesh] OR "Review"

[Publication Type] OR "Review Literature as Topic"[Mesh] )

"health equity " AND ("equity" OR "Inequality" OR "inequalities" OR "disparities") and * "Review"

\section{The inclusion criteria were}

- Literature review articles: Systematics or meta-analysis

- Articles that mention mechanisms (organization of the service, financing and participation or adjustment of services to the need), relationships, processes or ways in which equity in health is affected

- Articles with emphasis on the context of health policies: neoliberal orientation (privatization, need for regulation) or welfare states, with equality or targeting orientation 
- Articles that involve some aspect of: accessibility, opportunity, availability, acceptability, affordability, continuity, security, best practices in care, effectiveness, use of health resources, quality of health care and public health services

- Accessibility, Availability, Appropriateness, Acceptability, Continuity, Safety, Effectiveness, Utilization of Health promoting resources, Quality of care and Quality of Public Health services.

- Articles that include population groups of interest (geographical area, ethnic groups or specific health problem focused on from the health system) with interventions from the health system

- Quantitative or Qualitative Methodologies

\section{The exclusion criteria were:}

- Articles that focus on a disease or pathology only from a clinical or epidemiological aspect

- Articles that focus on socioeconomic factors without relating them to health care systems

- Articles with a focus on health sector workers unrelated to the impact of health services

- Articles that do not contain information for middle-income countries or that contain less than $33 \%$ of information for middle-income countries

\section{Conditions or domains to be studied}

Policies that affect equity in health with an impact on services or the health system.

\section{Participants / population}

Population of middle-income countries according to the World Bank classification for 2019: low middle and middle-high.

Intervention / exposure

Health equity

\section{Main outcomes}

resource transfer

contracting and payment mechanisms

decentralization of health services

barriers to accessing health services

\section{Effect measurements}

Does not apply

\section{Adicional outcomes}

accessibility, opportunity, availability, acceptability, affordability, continuity, security, best practices in care, effectiveness, use of health resources, quality of health care 


\section{Data extaction}

Data extraction matrix

Bias risk assestment

The texts that are finally included will be evaluated with PRISMA

Data synthesis strategy

CERQual

We will use The Confidence in the Evidence from Reviews of Qualitative research (CERQual)

Subgroups analysis

Does not apply

Aditional information contact

Fredy Rodríguez-Páez

Organización y afiliación del revisor

Reviewer filiation

Universidad Jorge Tadeo Lozano

Reviewer Team

Martha Riaño, Universidad Nacional de Colombia

Francisco Palencia, Pontificia Universidad Javeriana

Review type and method

Umbrella review

Start date: april /01/ 2020

Date ending: december / 15 / 2020

Grants

No

Conflict assert

We assert no have conflicto interest

Language

English

\section{Country}


Colombia

\section{Keywords}

Health Equity, inequalities, disparities, Delivery of Health Care, Middle Income Country 